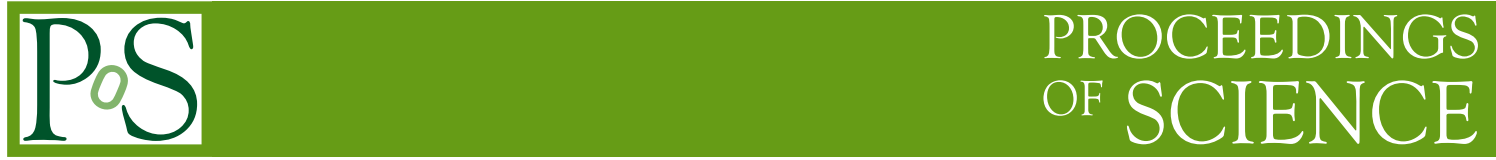

\title{
Top-quark production at the Tevatron and LHC
}

\section{Ulrich Langenfeld}

Julius-Maximilians-Universität Würzburg

E-mail: Ulrich.Langenfeld@physik.hu-berlin.de

\section{Sven-Olaf Moch}

Deutsches ElektronenSynchrotron, DESY

E-mail: sven-olaf.moch@desy.de

\section{Peter Uwer*}

Humboldt-Universität zu Berlin

E-mail: Peter.Uwer@Physik.HU-Berlin.de

We briefly review some recent progress in the theoretical description of top-quark pair production. In particular the cross section using the $\overline{\mathrm{MS}}$ mass is discussed together with the first direct determination of the running mass. We briefly comment also on a program published recently allowing the cross section evaluation using the running mass.

35th International Conference of High Energy Physics

July 22-28, 2010

Paris, France

\footnotetext{
*Speaker.
} 


\section{Introduction}

The top-quark is the heaviest fermion discovered so far. Therefore, it is a unique research topic. Due to its short lifetime it decays before it has time to form hadronic bound states [1]. Often this is summarized in the statement that the top-quark behaves as a quasi free quark. This allows precise theoretical predictions in perturbative QCD. As a consequence top-quark physics is an ideal laboratory for precise tests of the Standard Model (SM) at high energy scale. Topquark physics plays also an important role in searches for new physics. A necessary ingredient are precise theoretical predictions. A central quantity in that context is the total cross section. The experimental aim is to measure the total cross section with an accuracy of $\pm 5 \%$ [2]. Matching this accuracy with the theoretical predictions is a challenge which requires to take also small effects into account. In the following we summarize some recent results obtained in that direction. In the SM the only free parameter in top-quark physics - apart from the CKM matrix elements-is the top-quark mass. A precise knowledge of the top-quark mass is thus crucial for all analyses. Since the pole mass is not a well defined concept in QCD it is also important to study different mass definitions when aiming for high precision. In a recent study [3] the top-quark cross section in hadronic collisions has been evaluated for the first time using the mass in the modified minimal subtraction scheme $(\overline{\mathrm{MS}})$ often called the $\overline{\mathrm{MS}}$ mass or running mass. We will briefly review this analysis which has lead recently to the first direct determination of the $\overline{\mathrm{MS}}$ mass [3].

\section{Top-quark pair production in hadronic collisions}

We start by recalling the relevant formulae for the total cross section $\sigma_{p p \rightarrow t \bar{t} X}$ of top-quark hadro-production within perturbative QCD,

$$
\sigma_{p p \rightarrow t \bar{t} X}\left(S, m_{t}^{2}\right)=\sum_{i, j=q, \bar{q}, g} \int_{4 m_{t}^{2}}^{S} d s L_{i j}\left(s, S, \mu_{f}^{2}\right) \hat{\sigma}_{i j}\left(s, m_{t}^{2}, \mu_{f}^{2}\right)
$$

where $S$ denotes the hadronic center-of-mass energy squared, $m_{t}$ the pole mass of the top-quark and $\hat{\sigma}_{i j}$ the partonic cross sections. The standard definition for the parton luminosity $L_{i j}$ convolutes the two parton distribution functions (PDFs) $F_{i / p}$ at the factorization scale $\mu_{f}$ :

$$
L_{i j}\left(s, S, \mu_{f}^{2}\right)=\frac{1}{S} \int_{S}^{S} \frac{d \hat{s}}{\hat{s}} F_{i / p}\left(\frac{\hat{s}}{S}, \mu_{f}^{2}\right) F_{j / p}\left(\frac{s}{\hat{s}}, \mu_{f}^{2}\right),
$$

Note that due to the additional factor $1 / S$ the fluxes at the Tevatron and the LHC can be directly compared. From Eq. 2.1 we identify two sources for theoretical uncertainties in the cross section predictions: One is related to the partonic cross section in Eq. 2.1 while the other is due to uncertainties of the PDFs in Eq. 2.1 through the luminosity functions. At the Tevatron the PDFs are probed at larger $x$ values compared to the LHC. Furthermore the cross section at the Tevatron is dominated by quark-antiquark annihilation. As a consequence the PDF uncertainties at the Tevatron are almost twice as large as at the LHC [4] where the PDF uncertainty is of the order of 3-4\%. This statement is based on the error as provided by the PDF (for a detailed discussion we refer 
to [4]). An unpleasant feature currently observed is that different PDFs sets can lead to rather different predictions which are only marginally consistent when the PDF uncertainty is taken into account. This has been observed recently for example in a comparison between the MSTW2008 set [5] and the ABKM10 set [6]. The origin of this discrepancy is related to different $\alpha_{s}$ values used in the two sets and a different gluon fluxes at effective $x$ values about $2.5 \times 10^{-2}$. With increasing integrated luminosity at the LHC top-quark physics can be used to further constrain PDFs as suggested already in Ref. [7]. Note that in this context top-spin dependent observables may provide useful to disentangle the $g g$ contribution from the $q \bar{q}$ contribution. Below we briefly summarize some of the recent developments towards improved cross section predictions- the second source of possible uncertainties as stated before.

\section{Towards precise predictions for the cross section}

The QCD radiative corrections for the total cross section in Eq. 2.1 as an expansion in the strong coupling constant $\alpha_{s}$ are currently known completely at next-to-leading order (NLO) [8-11] and, as approximation, at next-to-next-to-leading order (NNLO) $[4,12]$. The latter result is based on the known threshold corrections to the partonic cross section $\hat{\sigma}_{i j}$, i.e. the complete tower of Sudakov logarithms in $\beta=\sqrt{1-4 m_{t}{ }^{2} / s}$ and the two-loop Coulomb corrections, i.e. powers $1 / \beta^{k}$ (see also [13] for some recent improvements). It also includes the complete dependence on $\mu_{f}$ and the renormalization scale $\mu_{r}$, both being known from a renormalization group analysis. The presently available perturbative corrections through NNLO lead to accurate predictions for the total hadronic cross section of top-quark pairs with a small associated theoretical uncertainty $[3,4,12]$ (see also e.g. [14] for related theory improvements through threshold resummation). For further refinements studied recently we refer to [13,15-18].

Aiming for a precision of the theoretical predictions at the per cent level also electroweak contributions need to be taken into account. At the LHC these correction can amount up to 1$2 \%$, for details we refer to Refs. [19-21]. At the Tevatron the weak effects are only about $0.2 \%$ for reasonable Higgs masses. The different behavior at the LHC compared to the Tevatron is a consequence of the different collider energies. Naively one would expect the weak corrections to be small owing to the smallness of the coupling constant. However at high energies Sudakov logarithms can enhance the weak corrections. Since the Sudakov logarithms could be canceled at least partially by including real gauge boson production this effect gives a negative contribution to the cross section. Due to the limited collider energy this effect gives only a small correction at the Tevatron. At the LHC events with high momentum transfer appear more frequently and the effect is thus more important compared to the Tevatron. While for the total cross section the correction amounts to a few percent only we stress that for differential distributions at high momentum transfer they can be of the order of 10\% [19-21]. Since this is also the region where new physics effects from heavy resonance may appear it is important to take the weak corrections into account.

Very close to the threshold the attractive part of the QCD potential may lead to remnants of a would be bound state $[22,23]$. These corrections affect significantly differential distributions in the threshold region. A prominent example is the $m_{t t}$-distribution, the invariant mass distribution of the top-quark pair. Due to bound state effects the differential cross section obtains also a contribution from kinematic regions below the nominal production threshold. If one could resolve this region 
experimentally it would provide a sensitive method to measure the top-quark mass similar to what is proposed for a future $e^{+} e^{-}$linear collider. The correction of the total cross section due to this effect is of the order of $10 \mathrm{pb}$ at the LHC with $\sqrt{S}=14 \mathrm{TeV}$. At the Tevatron where color octet production dominates this effect is less important.
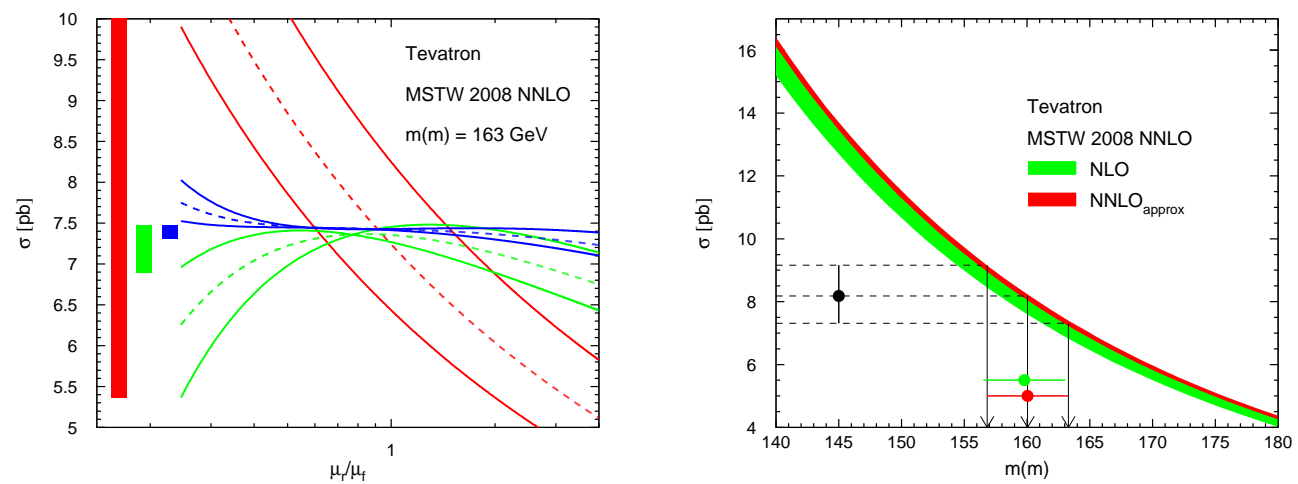

Figure 1: The total cross section using the $\overline{\mathrm{MS}}$ mass (left). The determination of the $\overline{\mathrm{MS}}$ mass (right).

It is known that the pole mass of a quark is not a well defined concept in QCD [24, 25]. As a consequence the pole mass has an intrinsic uncertainty of the order of $\Lambda_{\mathrm{QCD}}$. Aiming for mass measurements with an accuracy of $1 \mathrm{GeV}$ or even below it is clear that the use of the pole mass is questionable. An alternative idea to the currently pursued measurements at the Tevatron could be to recalculate the total cross section using the $\overline{\mathrm{MS}}$ mass instead of the pole mass. From the comparison of the measured cross sections with the theoretical predictions a direct determination of the $\overline{\mathrm{MS}}$ mass is possible. This has been done recently for the first time in Ref. [3]. At the Tevatron, the use of the $\overline{\mathrm{MS}}$ mass leads to an improved behavior of the perturbative expansion compared to the pole mass. In Fig. 1 the different orders in perturbation theory that is leading-order, next-to-leading order and the approximation to NNLO are shown using the $\overline{\mathrm{MS}}$ mass. We observe that the NLO curve and the approximation to NNLO are very close to each other showing the aforementioned improvement. Due to the improved convergence the extracted values of the top-quark running mass are very stable with respect to different orders. The determination is illustrated in Fig. 1 and the results are shown in Tab. 1.

\begin{tabular}{c|c|c|c} 
& LO & NLO & NNLO $_{\text {approx }}$ \\
\hline $\bar{m}(\bar{m})$ & $159.2_{-3.4}^{+3.5}$ & $159.8_{-3.3}^{+3.3}$ & $160.0_{-3.2}^{+3.3}$
\end{tabular}

Table 1: Determination of the running mass from cross section measurements.

\section{HATHOR - HAdronic Top and Heavy crOss section calculatoR}

The aforementioned theoretical progress has to be made accessible to the experimental analysis. Recently a program has been published including the QCD corrections mentioned before [26]. 
In particular it is possible to chose between different approximations at the NNLO level. In addition the program allows the direct evaluation of the cross section using the $\overline{\mathrm{MS}}$ mass. The package includes a small library so that the results can be easily used in the experimental analysis. It is foreseen to include the weak corrections in the next release.

Acknowledgments: This work is supported in part by the Deutsche Forschungsgemeinschaft through the Transregional Collaborative Research Centre SFB-TR9 "Computational Particle Physics" and the Helmholtz Alliance "Physics at the Terascale" contract VH-HA-101.

\section{References}

[1] I. I. Y. Bigi, Y. L. Dokshitzer, V. A. Khoze, J. H. Kuhn, and P. M. Zerwas, Phys. Lett. B181, 157 (1986).

[2] G. Aad et al., "Expected Performance of the ATLAS Experiment - Detector, Trigger and Physics", by the ATLAS Collaboration. Jan 2009, e-Print: arXiv:0901.0512 [hep-ex].

[3] U. Langenfeld, S. Moch, and P. Uwer, Phys. Rev. D80, 054009 (2009).

[4] S. Moch and P. Uwer, Phys. Rev. D78, 034003 (2008).

[5] A. D. Martin, W. J. Stirling, R. S. Thorne, and G. Watt, Eur. Phys. J. C63, 189 (2009).

[6] S. Alekhin, J. Blumlein, S. Klein, and S. Moch, Phys. Rev. D81, 014032 (2010).

[7] W. Bernreuther, A. Brandenburg, Z. G. Si, and P. Uwer, Phys. Rev. Lett. 87, 242002 (2001).

[8] P. Nason, S. Dawson, and R. K. Ellis, Nucl. Phys. B303, 607 (1988).

[9] W. Beenakker, H. Kuijf, W. L. van Neerven, and J. Smith, Phys. Rev. D40, 54 (1989).

[10] W. Bernreuther, A. Brandenburg, Z. G. Si, and P. Uwer, Nucl. Phys. B690, 81 (2004).

[11] M. Czakon and A. Mitov, Nucl. Phys. B824, 111 (2010).

[12] S. Moch and P. Uwer, Nucl. Phys. Proc. Suppl. 183, 75 (2008).

[13] M. Beneke, M. Czakon, P. Falgari, A. Mitov, and C. Schwinn, Phys.Lett. B690 483 (2010),

[14] M. Cacciari, S. Frixione, M. L. Mangano, P. Nason, and G. Ridolfi, JHEP 09, 127 (2008).

[15] M. Beneke, P. Falgari, and C. Schwinn, Nucl. Phys. B828 69 (2010).

[16] M. Beneke, P. Falgari, and C. Schwinn, Nucl. Phys. B842 414 (2010).

[17] V. Ahrens, A. Ferroglia, M. Neubert, B. D. Pecjak, and L. L. Yang, Phys. Lett. B687 331 (2010).

[18] V. Ahrens, A. Ferroglia, M. Neubert, B. D. Pecjak, and L. L. Yang, JHEP 1009097 (2010).

[19] W. Beenakker et al., Nucl. Phys. B411 343 (1994).

[20] J. H. Kuhn, A. Scharf, and P. Uwer, Eur. Phys. J. C51 37 (2007).

[21] W. Bernreuther, M. Fucker, and Z.-G. Si, Phys. Rev. D78 017503 (2008).

[22] K. Hagiwara, Y. Sumino, and H. Yokoya, Phys. Lett. B666 71 (2008).

[23] Y. Kiyo, J. H. Kuhn, S. Moch, M. Steinhauser, and P. Uwer, Eur. Phys. J. C60 375 (2009).

[24] I. I. Y. Bigi, M. A. Shifman, N. G. Uraltsev, and A. I. Vainshtein, Phys. Rev. D50 2234 (1994).

[25] M. Beneke and V. M. Braun, Nucl. Phys. B426 301 (1994).

[26] M. Aliev, H. Lacker, U. Langenfeld, S. Moch, P. Uwer and M. Wiedermann, “- HATHOR HAdronic Top and Heavy quarks crOss section calculatoR”, arXiv:1007.1327, to appear in CPC. 